\title{
Bhabha Jacqueline, Child Migration and Human Rights in a Global Age
}

Bénédicte Lavaud-Legendre

\section{(2) OpenEdition}

Journals

Édition électronique

URL : https://journals.openedition.org/remi/7848

DOI : $10.4000 /$ remi. 7848

ISSN : $1777-5418$

Éditeur

Université de Poitiers

Édition imprimée

Date de publication : 1 septembre 2016

Pagination : 169-170

ISBN : 979-10-90426-27-6

ISSN : 0765-0752

Référence électronique

Bénédicte Lavaud-Legendre, "Bhabha Jacqueline, Child Migration and Human Rights in a Global Age », Revue européenne des migrations internationales [En ligne], vol. 32 - n² | 2016, mis en ligne le 01 septembre 2016, consulté le 16 avril 2022. URL : http://journals.openedition.org/remi/7848 ; DOI : https://doi.org/10.4000/remi.7848 


\section{Notes de lecture}

Bhabha, Jacqueline

Child Migration and Human Rights in a

Global Age. - Princeton ; Oxford : Princeton

University Press, 2014. - $374 \mathrm{p}$.

ISBN : 978-0-691-14360-6

Dans son ouvrage intitulé Child Migration and Human Rights in a Global Age, Jacqueline Bhabha, directrice de recherche au centre François-Xavier Bagnoud pour la Santé et les Droits de l'Homme, à l'université d'Harvard, se livre à un diagnostic sans concession quant à la manière dont les mineurs migrants, bien souvent séparés de leurs parents, sont considérés et accompagnés durant leur développement dans différents pays du monde. Elle identifie ainsi l'incroyable ambivalence de nos sociétés. Considérés à la fois comme devant être protégés, parce que vulnérables, ces mineurs sont dans le même temps condamnés comme coupables d'infractions à la législation et à ce titre, écartés des dispositifs de protection de l'enfance ; mineurs auxquels on reconnaît le droit à une vie familiale normale, c'est-à-dire le droit de grandir entourés de ses deux parents, alors que dans le même temps les politiques migratoires ont bien souvent pour effet de les séparer de leurs parents ; mineurs auxquels on reconnaît le droit à l'éducation, alors que dans le même temps les délais de prise en charge font obstacle à leur accès à la scolarité ; mineurs auxquels on reconnaît une certaine autonomie et une volonté propre, alors que dans le même temps de telles qualités sont bien souvent ignorées par les dispositifs d'accompagnement. Pour ne retenir qu'un exemple, I'auteur souligne que les enfants nés sur le sol américain ont le droit de rester y vivre, mais ce droit ne s'applique pas à leurs parents. II n'est donc pas rare que des parents puissent être renvoyés en faisant éventuellement le choix de laisser leurs enfants lorsqu'ils sont convaincus qu'ils n'auront pas d'avenir possible dans leur pays d'origine. Retenant une approche globale, elle analyse la réponse des Cours européennes en présence d'une difficulté comparable. La Cour européenne des droits de l'homme ne se réfère pas à l'intérêt des mineurs, mais confronte uniquement l'intérêt des parents à ceux de l'État. À l'inverse, la Cour de justice de I'Union européenne inclut dans son appréciation l'intérêt des enfants.

En étayant sa démonstration par de nombreux exemples extrêmement documentés, Jacqueline Bhabha aborde différents contextes de migration : le rapport à la famille (ce qui regroupe le droit à vivre avec ses parents, l'épineuse question de l'accès à la citoyenneté et l'adoption) ; l'exploitation (dans le cadre de la traite des êtres humains ou sous l'angle spécifique des enfants soldats) et enfin, la migration comme mode de survie notamment suite au printemps arabe. L'ambivalence identifiée se décline dans l'ensemble des champs abordés. Elle se traduit par la complexité des procédures, l'absence d'engagement de bon nombre d'acteurs auprès de ces jeunes et évidemment l'insuffisance des moyens dédiés. II en découle de conséquentes violations des droits de ces mineurs, de ces droits-mêmes qui sont explicitement proclamés par les textes internationaux. On peut croire qu'une clarification des objectifs de nos sociétés permettrait de résoudre bon nombre de difficultés, à droits et à moyens constants. II est pour cela nécessaire d'oser définir un certain nombre de priorités. Jacqueline Bhabha en définit plusieurs : l'accès des mineurs à un statut légal, à des droits 
économiques et sociaux et à un environnement social protecteur, ce qui implique dans la plupart des cas, le maintien des liens avec les parents. Respecter les droits des mineurs migrants, c'est également considérer leur aspiration à exprimer une volonté propre, à avoir un projet professionnel, un projet de vie. Les infantiliser ou les considérer uniquement sous l'angle répressif est bien souvent contre-productif. Leur reconnaître des droits durant leur minorité est la condition sine qua non pour qu'ils puissent assumer les obligations qui seront les leurs une fois majeurs. Ils ne seront les forces vives de notre monde de demain que si on leur en donne les moyens.

Bénédicte Lavaud-Legendre Chargée de recherche CNRS, juriste COMPTRASEC/Université de Bordeaux

Armagnague-Roucher, Maïtena

Une jeunesse turque en France et en

Allemagne. - Lormont : Le Bord de l'eau, 2016. $-269 \mathrm{p}$.

ISBN : 978-2-35687-433-7

L'ouvrage de Maïtena ArmagnagueRoucher est issu d'une thèse de sociologie sur les jeunesses d'ascendance turque vivant en France et en Allemagne et, plus précisément, à Bordeaux et à Hambourg. Le pari de comparer une immigration minoritaire en France à une immigration bien plus nombreuse et plus ancienne en Allemagne était osé, mais l'auteure a brillamment réussi l'exercice en multipliant les jeux d'échelle et de comparaison entre le local, le national et le transnational. Cette fine compréhension témoigne également d'une grande sensibilité et capacité d'écoute se donnant à voir dans les nombreux extraits d'entretiens qui lui ont permis de recueillir des récits d'expériences parfois très intimes.

Le premier chapitre présente le cadre national, institutionnel et migratoire dans lequel s'inscrivent les jeunesses turques dans les deux pays d'immigration. II s'ouvre sur un bref rappel du débat politique lié à la question récurrente de l'intégration des migrants en provenance de Turquie et de leurs enfants, où I'on constate que les fantasmes politiques et médiatiques croisent parfois, des deux côtés du Rhin, une certaine rhétorique sur I'" exception turque " portée par des démographes quelque peu oublieux des conjonctures historiques, économiques et sociologiques. Surtout, la fausse évidence de deux systèmes politiques d'intégration différents est battue en brèche : l'auteure montre que si les conceptions française et allemande de la citoyenneté sont différentes, ces modèles ne sont pas pour autant opposés, rappelant en cela l'ouvrage coordonné par Bertossi (2007) sur les politiques de citoyenneté en France et en Grande-Bretagne.

Les deux chapitres suivants concernent la participation communautaire, entendue comme une autonomie vis-à-vis de la société majoritaire, le désir de rester entre soi. En France, Maïtena Armagnague montre que celle-ci touche plutôt les domaines socio-économique, socioculturel et scolaire. À Bordeaux, l'entrepreneuriat turc, principalement dans le secteur du bâtiment, constitue un apprentissage de la vie pour les jeunes qui sont employés dans les entreprises familiales avant de créer la leur propre. Loin de dénoncer ces liens communautaires, la sociologue souligne que ces expériences représentent un mode à part entière de socialisation et, de fait, permettent aux jeunes de s'insérer économiquement et par l'emploi. Sans compter que cette intégration communautaire peut être une réponse à une conjoncture économique parfois difficile, ainsi qu'au racisme au travail - bien que l'auteure ne s'attarde pas sur ce dernier point. Du point de vue des relations amicales et amoureuses, les jeunes interrogés disent privilégier 
les relations entre Turcs musulmans dès lors que les enjeux sont sérieux, comme celui du mariage, ou récurrents, comme les types de sociabilité en banlieue. En centre-ville, comme en périphérie urbaine, il est peu question de fréquenter durablement les "Français " ni même les "Arabes ". Pour les jeunes hommes, la communauté ethnique fonctionnerait ainsi comme un contrôle juvénile, un antidote de la " galère " et des dérives des jeunes dans les périphéries urbaines. Du côté des filles, les rôles sociaux sont, sans surprise, davantage tournés vers la sphère familiale et domestique. Toutefois, au-delà de discours juvéniles assez convenus, l'auteure sait déceler des indices d'un accommodement de la part de ces jeunes, des ajustements entre ces pratiques de sociabilité et la conformité à l'ordre moral communautaire. Enfin, l'école, à travers une inscription majoritaire dans les filières professionnelles, permet de maîtriser un secteur d'expertise et un métier, souvent en lien avec l'activité familiale. Plutôt que facteur d'ascension sociale, l'école sert à ces jeunes d'outil à une modalité de participation sociale passant par la communauté, comme cela a été souligné avec l'emploi.

La force du troisième chapitre réside dans sa volonté de mesurer l'effectivité de l'a priori selon lequel les figures d'intégration par la communauté semblent favorisées en Allemagne du fait de la tradition sociologique qui envisage la communauté comme un espace social structurant (voir Gemeinschaft de Tönnies, 2010 [1887]). Maïtena Armagnague propose des indicateurs qualitatifs de mesure à travers les expériences sociales des jeunes originaires de Hambourg. En Allemagne, les populations turques sont parfois désignées - tant par la société que par une partie d'entre elles - comme entité homogène unifiée, niant de ce fait l'importante diversité culturelle, religieuse, linguistique et politique, accréditant même la thèse de la constitution d'une société parallèle (Parallelgesellschaft). Cette représentation est mise à mal par la sociologue qui montre que les solidarités ethniques peuvent s'éroder à mesure de l'installation durable des Turcs en Allemagne ; celle-ci produisant un processus d'acculturation : la pratique de la langue turque s'affaiblit, les modes de consommation, d'épargne et d'insertion professionnelle s'éloignent de ceux des aînés, les jeunes tendent à adopter les comportements et les aspirations économiques des Allemands de la classe moyenne. Si les restitutions d'entretiens montrent que la figure d'intégration communautaire est ambivalente, oscillant entre l'entre-soi et l'expérience d'acculturation, I'analyse globale des indicateurs qualitatifs d'intégration montre que la construction de la " minorité majoritaire " des Turcs en Allemagne est bien différente de celle de la " minorité minoritaire " en France.

Le chapitre suivant s'attarde fort opportunément sur cette condition minoritaire dont les différentes expressions, ethnicisées ou religieuses, interrogent les principes démocratiques des sociétés française et allemande. Partant de l'hypothèse que la promesse, individualiste et égalisatrice, fondatrice de ces deux sociétés ne se concrétise pas pour la jeunesse originaire deTurquie, l'auteure avance que l'impasse de cette situation crée un univers de revendications liées à l'espace urbain, à I'accès au système scolaire et à l'emploi, à la sexualité. En dépit de politiques migratoires et d'accueil différentes dans les deux pays, ainsi que d'une acception différenciée du fait minoritaire et/ou communautaire, Maïtena Armagnague peint un sombre tableau où les minorisés seraient " passés d'une culture de la résistance à une culture oppositionnelle " comme conséquence des injustices et des inégalités sociales vécues ou perçues par ces populations juvéniles des deux côtés du Rhin.

Toutefois, le chapitre cinq vient tout à la fois approfondir et nuancer ce constat 
en précisant que l'expérience minoritaire fait écho à des contextes urbains, interethniques et institutionnels distincts qui lui donnent une forme différente dans ses modes d'expression : plus démonstratives et contestataires en France, plus citoyennes en Allemagne. II en ressort selon l'auteure deux formes de conditions minoritaires : communauté " marginale " en France où la société est vue comme "discriminante ", et communauté " nationale " en Allemagne où elle est considérée comme " racialisée ". Marginale en France parce que construite à partir de l'amertume d'être cantonnés aux marges de la société majoritaire. En ce sens, la jeunesse d'ascendance turque se rapprocherait de la catégorie des "jeunes de banlieue " et se réapproprierait une rhétorique victimaire attachée à la domination coloniale et postcoloniale. Outre-Rhin, les rapports aux institutions majoritaires seraient moins tendus, alors que les enclaves urbaines produisent pourtant davatange de situations objectives de marginalité. Même si l'Allemagne a longtemps refusé de s'accepter comme pays d'immigration, sa plus grande ouverture à la multiculturalité (Multikulti) et son refus de l'assimilationnisme offriraient aux jeunes de développer des tentatives expérimentales de lutte contre la marginalisation, sans pour autant leur donner le sentiment d'être membres d'une " communauté de citoyens ". Et l'auteure de conclure qu'« il y a donc corrélation entre les philosophies des "modèles" et leurs principes subjectifs expérientiels d'exclusion ".

L'ouvrage montre également que tous les parcours ne sont bien sûr pas linéaires ni ne s'apparentent au schéma minoritaire et que des mobilités sociales individuelles ascendantes passent par la mise à distance de la communauté et de la marginalité urbaine. Souvent valorisées par les institutions dominantes en raison de l'assimilation culturelle et de l'intégra- tion économique dont elle est le reflet, parfois dénoncées par les sciences sociales lorsque ces "figures de la réussite " sont médiatisées sans que l'on s'interroge a contrario sur les processus d'exclusion/ inclusion (Rigouste, 2007), ces mobilités ascendantes entrent en conformité avec les normes sociales, économiques et symboliques des classes moyennes comme le montre l'avant-dernier chapitre. Loin de céder aux clichés de la réussite, Maïtena Armagnague sait montrer les paradoxes d'expériences qui n'en sont parfois pas moins douloureuses. Car ces parcours de réussite scolaire, sociale et/ou économique peuvent s'opérer au prix d'un éloignement des espaces ethniques de socialisation, voire de leur famille, ce qui n'est pas sans provoquer des « déchirures ".

Enfin, le dernier chapitre confronte les modèles nationaux d'intégration à la structuration en communauté transnationale des jeunes d'ascendance turque. L'auteure $y$ décrit des phénomènes originaux et assez inattendus, en particulier celui d'une intégration par la communauté dans le cas français et d'un délitement progressif de la communauté en Allemagne. En dépit de dynamiques communes entre les deux pays, eu égard notamment aux processus de relégation sociale, le traitement des inégalités sociales diverge, ce qui conduit l'auteure à poser plus largement en guise d'ouverture la question du devenir des jeunesses de France et d'Allemagne, au-delà du strict cas des jeunes d'ascendance turque ou même immigrée.

L'ouvrage de Maïtena Armagnague constitue un apport considérable à la compréhension des sociétés migratoires contemporaines, bien au-delà du strict cas des jeunesses d'ascendance turque. Si I'on peut regretter le peu de cas porté à la diversité des composantes ethniques, linguistiques et religieuses de cette population, l'une des principales forces de ce livre est de construire un problème 
sociologique qui, finalement, parle autant des minoritaires que des majoritaires et apporte de précieuses clés de compréhension sur le fonctionnement des sociétés contemporaines.

\section{..: Références bibliographiques}

Bertossi Christophe (Ed.) (2007) European Anti-Discrimination and the Politics of Citizenship. Britain and France, New York, Palgrave Macmillan, 246 p.

Rigouste Mathieu (2007) La construction médiatique du corps intégré. Promouvoir pour bannir dans la cité postcoloniale, in Isabelle Rigoni Éd., Qui a peur de la télévision en couleurs ? La diversité culturelle dans les médias, Montreuil, Aux Lieux d'Être, pp. 109-129.

Tönnies Ferdinand (2010 [1887]) Communauté et société, Paris, PUF, $336 \mathrm{p}$.

Isabelle Rigoni

Chercheure, Centre Émile Durkheim

(UMR-CNRS, Bordeaux)

Maître de conférences en sociologie, INS

HEA Suresnes

Merckaert, Alain (dir.)

Des femmes plurielles. - Amiens : Licorne,

2013. - 207 p.

ISBN : 978-2-910449-42-1

L'ouvrage d'Alain Merckaert intitulé Des Femmes Plurielles est lui-même pluriel dans sa forme et dans son contenu. Y sont dressés les portraits de douze femmes venues de pays aussi différents que la Géorgie, le Gabon ou Haïti, pour ne citer que ceux-là, afin de s'installer dans le Nord de la France. Au travers de ces portraits se dessinent des trajectoires qui partagent un point commun, celui d'une vie citoyenne faite d'engagements dans le tissu associatif et politique local en Picardie et en Normandie.

Les récits de vie sont contés un à un, fruits d'un travail sociologique d'entretiens dans un premier temps et d'un travail d'écri- ture littéraire réalisé dans un second temps par cinq auteures : Odile Glinel, Isabel Asunsolo, Dominique Marie Godfard, Léo Lamarche et Mirita Ribeiro. Ainsi, la singularité et la richesse des parcours viennent se refléter dans la diversité des formes d'écriture employées (récit à la première ou à la troisième personne, extraits de journal, abécédaire) et des styles littéraires de chacune des auteures. L'ouvrage entend partager avec les lecteurs des histoires à la fois ordinaires, par leur ancrage dans le quotidien, et exceptionnelles, par la force qui se dégage de chacun des récits. L'objectif poursuivi par le directeur de la publication au travers de cette démarche est de partager ces témoignages avec le plus grand nombre.

Si les parcours sont singuliers et les expériences individuelles, émergent au sein de cet ouvrage plusieurs thèmes transversaux : d'une part, les questions qui ont été au cœur des entretiens (" leur vie en tant que femme, en tant que personne traversée par une diversité culturelle ; et en tant que citoyenne active ") et d'autre part, des expériences partagées dans les étapes de la migration telles que les questions de l'adaptation, de la relation au pays d'origine, ou encore de la transmission de la culture aux enfants.

En premier lieu, de ces douze parcours de femmes émerge une force d'agir et de façonner son propre destin. Femmes immigrées ou enfants d'immigrés, ce ne sont pas les obstacles à surmonter qui manquent, mais chacun des parcours illustre comment le parcours migratoire, le leur ou bien celui de leurs parents, leur procure une forte détermination et leur permet de surmonter les difficultés initiales afin de mener une vie indépendante. Habiba, originaire d'Essaouira au Maroc, refuse de se conformer à une vie conjugale qu'elle n'a pas choisie et part avec sa fille à Agadir puis en France où elle va créer une entreprise de formation quelques années plus tard. Seela, Mauricienne, travaille un 
an dans une usine pour économiser un pécule en vue de son départ vers la France où elle deviendra cadre administratif à la Délégation régionale aux droits des femmes. Anatolie, quant à elle, obtient une bourse au Rwanda pour étudier en France, part en faisant la fierté de son père et devient professeur d'histoire-géographie. Ces trois exemples sont à l'image de l'ouvrage : des pays et des milieux d'origine très différents, mais un fil conducteur à trouver dans la détermination de ces femmes à décider du cours de leur vie.

Ensuite, ces parcours sont imprégnés de ce qu'Alain Mackaert nomme " être traversé par une diversité culturelle ". Ces cultures constituent des ressources précieuses dans la vie de ces femmes et forgent leurs engagements. Lika, arrivée en France de Géorgie en tant que réfugiée politique avec son fils âgé de quelques mois à peine, son mari et sa mère, ouvre un restaurant géorgien après avoir obtenu un CAP cuisine. Samia, née dans une famille marocaine berbère, crée dans un quartier d'Amiens une association devenue incontournable dans la vie locale. Chacun des récits illustre ainsi à sa manière comment des parcours individuels donnent corps à une société plurielle, comment les actions de chacune viennent enrichir nos expériences collectives et faire vivre la diversité, un concept dont il est souvent question de manière abstraite et qui se trouve ici incarné.

Toutes les femmes que nous rencontrons au fil de la lecture sont par ailleurs engagées et militantes, que ce soit dans la sphère professionnelle, associative ou politique. Si les causes qui leur sont chères sont parfois ancrées dans les expériences vécues lors du parcours migratoire et de l'arrivée dans un nouveau pays, les engagements sont multiples et ne sauraient être réduits à cette dimension. Mireille, venue du Gabon, est volontaire dans une crèche et milite auprès des sans-papiers jusqu'à ce qu'elle fonde sa propre association avec le projet de créer une crèche parentale et d'ouvrir un restaurant africain. Jola, qui a quitté la Pologne peu après la chute du régime communiste, s'est quant à elle engagée corps et âme pour les causes écologiques, notamment contre le nucléaire.

Au-delà des trois thèmes qui font cet ouvrage, la richesse des récits évoque de nombreuses autres questions. Tout d'abord, celle des ajustements que la migration appelle nécessairement. Zohra partie du Maroc à dix-huit ans, préfère par exemple le terme " adaptation " à celui d'" intégration " à la tonalité trop assimilationniste. La relation au pays d'origine constitue un autre sujet de taille. Maria, née en France de parents portugais, envisage ainsi de prendre en charge la ferme familiale au Portugal dans le cadre d'un éventuel projet de retour. Enfin, et sans que ces trois points ne soient exhaustifs, se pose la question de la transmission de la culture et de la langue des parents aux enfants. Anatolie, venue du Rwanda, souhaiterait que ces enfants parlent le kinyarwanda et puissent connaître les contes de sa mère, dont le souvenir s'efface déjà.

Cet ouvrage collectif parvient à rendre l'épaisseur des histoires individuelles, tout en faisant émerger des figures au travers de ces portraits singuliers. En écrivant l'histoire de Habiba, l'auteure Isabel Asunsolo mentionne par exemple ses origines espagnoles, tant certains vécus lui semblent familiers. Les récits partagés par ces douze femmes résonnent au-delà de l'expérience individuelle. Ces portraits se dessinent dans l'entrelacement de subjectivités : celle des femmes qui participent à cette aventure, celle des auteures qui rencontrent ces femmes et travaillent le texte à leur manière et enfin, celle des lecteurs qui se font le réceptacle de ces récits. Si cet ouvrage se rapproche davantage de l'œuvre littéraire que d'un travail sociologique, la richesse 
du matériau à partir duquel ces histoires sont écrites est remarquable et l'objectif de l'ouvrage annoncé dès les premières lignes, " donner une forme littéraire à ces témoignages, de façon à les porter vers le public " est indéniablement réussi.

Pour conclure, il convient de reconnaître que l'entreprise littéraire originale de cet ouvrage permet d'accorder une place de " sujet " et non $d^{\prime}$ " objet " aux participantes, évitant ainsi l'objectivisation qu'opère parfois l'analyse sociologique quelque peu hâtivement. Les voix des femmes prennent ici toute leur place, elles sont mises à l'honneur dans la méthodologie (relecture du travail littéraire produit à partir des entretiens) ainsi que dans le texte final structuré autour des témoignages individuels. Cette forme d'écriture représente ainsi un bel hommage à l'engagement de ces femmes qui se nourrit de leur vécu et de leurs expériences.

Nina Sahraoui Research Associate Robert Schuman Centre for Advanced Studies/European University Institute

\section{Diaz, Delphine}

Un asile pour tous les peuples? Exilés et réfugiés étrangers en France au cours du premier XIXe siècle. - Paris : A. Colin, 2014. $-316 \mathrm{p}$.

ISBN : 978-2-200-28927-0

Dans la première moitié du XIXe siècle, la France accueillit de nombreux étrangers ayant dû quitter leur pays pour des raisons politiques. Delphine Diaz a consacré sa thèse à ce phénomène et en publie une version probablement allégée. Elle rappelle d'abord que, depuis l'Ancien Régime et surtout la constitution de 1793, la France perpétue la tradition de l'accueil, poursuivie sous le Premier Empire, la Restauration, la monarchie de Juillet et la Deuxième République. À cette époque aucune définition juridique de l'exil politique n'existe, ce qui explique le caractère flou du vocabulaire alors utilisé : on parle indifféremment de "proscrits ", d'u exilés ", de " réfugiés ", voire de "bannis ", que les personnes concernées soient parties volontairement pour se mettre à l'abri ou qu'elles aient été contraintes au départ. Les distinctions entre exilés et immigrés du travail ou même touristes n'apparaissent pas encore nettement. Le dénombrement des étrangers ne peut être effectué, car ces derniers sont recensés en tant que tels seulement à partir de 1851. En fait la nationalité ne constitue pas encore une réalité juridique précisément définie. Aussi doit-on admettre de larges fourchettes statistiques. Les premiers arrivants sont des "Égyptiens ", en fait des soldats originaires des pays méditerranéens venus après la campagne de Bonaparte en Égypte. La première vague importante d'arrivées comprend 10000 à 12000 Espagnols qui avaient suivi Joseph Bonaparte, placé par son frère sur le trône de Madrid. Puis, en 1823, entrent en France des libéraux espagnols hostiles à Ferdinand VII, suivis par des Espagnols légitimistes carlistes à partir de 1833. Dans les années 1820 affluent également des patriotes allemands et grecs ainsi qu'1 millier de libéraux portugais. Les Polonais deviennent majoritaires et constituent $87 \%$ des réfugiés secourus en 1835. Chez les Russes de distinguent des libéraux comme Nicolas Tourgueniev et des révolutionnaires comme Bakounine et Herzen. Après la révolution de février 1848, la France devient une destination reine pour les exilés de toutes origines : les demandes de secours passent de 8000 en 1847 à 12000 à la fin de 1848.

Sur le plan administratif, la monarchie de Juillet est réputée avoir mis au point un statut en faveur des réfugiés. En vérité, cette réputation est imméritée, car les pratiques d'accueil furent expérimentées à la fin de l'Ancien Régime avec la constitution de dépôts-logements, la distribution de subsides mensuels, la distinction établie 
entre civils et militaires. Sous la restauration, l'accueil est inspiré par les principes de la charité chrétienne ; sous la monarchie de Juillet, cette conception ne disparaît pas totalement, mais elle se trouve de plus en plus relayée par une gestion administrative de la question. Les droits et devoirs des bénéficiaires sont progressivement définis, une attitude réservée leur est demandée, les immigrés du travail sont écartés du système de secours. L'attribution de ces derniers permet de bien surveiller les communautés étrangères. Avec la Seconde République, les contraintes s'allègent, l'accès à la naturalisation s'assouplit, la liberté de circulation sur le territoire français est offerte. Mais, très vite, la rigueur revient et est encore renforcée après le coup d'État de LouisNapoléon Bonaparte du 2 décembre 1851.

L'entrée en France se fait généralement par voie terrestre, souvent à pied : le trajet depuis Varsovie prend une trentaine de jours. Les méridionaux, Grecs et Italiens, viennent plutôt par la voie maritime. Les arrivants doivent posséder un passeport octroyé par un consul français ; cependant beaucoup de faux documents sont en circulation. L'installation à Paris se révèle plutôt exceptionnelle et est réservée aux plus fortunés ou aux élites intellectuelles comme Heine, Marx, Mickiewicz. La règle générale est l'assignation à résidence dans les dépôts de province ; les réfugiés ayant des ressources peuvent être autorisés à se loger à leurs frais. Le mélange des nationalités dans les dépôts est évité. À partir de 1832, les grands dépôts tendent à disparaître et, en 1848, l'accès à Paris devient libre, décision vite rapportée. Quant à devenir Français, malgré l'assouplissement et la création d'une procédure plus légère appelée admission à domicile, les candidats restent peu nombreux, par crainte de trahir leur idéal national : seulement vingt Polonais sur les 4972 bénéficiant de subsides officiels en 1837. Beaucoup de réfugiés ont laissé leur famille au pays et espèrent la faire venir. Aussi souhaitent-ils travailler, mais la barrière linguistique se révèle redoutable et, parfois, les réfugiés se heurtent à l'hostilité des Français qui redoutent la concurrence. Les emplois occupés sont très divers ; l'enquête de 1839 relève 120 métiers allant, par ordre alphabétique, des accordeurs de pianos aux agents voyers. Les militaires peuvent s'engager dans la Légion étrangère créée en 1831 et réorganisée en 1835. Les étudiants se dirigent surtout vers la médecine. Le Collège de France offre deux chaires, à I'Italien Pellegrino Rossi et au Polonais Adam Mickiewicz. La majorité des réfugiés est catholique, ce qui facilite l'insertion sociale, mais les minorités orthodoxe, protestante et juive ne sont pas négligeables. Les Polonais sont les plus attachés à la préservation de leur culture et à cet effet fondent une école en 1842.

Les déplacements des réfugiés sont nombreux, surtout entre la GrandeBretagne, la Suisse, la Belgique, parfois le Nouveau Monde et la France qui, souvent, constitue seulement une étape. Le gouvernement $n$ 'hésite pas à expulser les individus jugés indésirables, comme Mazzini en 1832. Ceux qui restent vivent dans une sociabilité spécifique. Beaucoup fréquentent les salons, notamment chez le marquis de La Fayette et la princesse Belgiojoso. Très appréciés sont aussi les cafés, les cabinets de lecture, les salles de spectacles où I'on s'initie aussi à la vie française. Les cortèges funéraires et les banquets, accompagnés de discours, sont propices à l'exposé des idées, surtout pour les libéraux. Les Français fondent des comités caritatifs ou politiques pour défendre les exilés; les premiers de ces comités sont philhellènes ; puis apparaît en 1831 le Comité central français en faveur des Polonais impliqués dans le soulèvement de Varsovie. Malgré la loi interdisant, sauf autorisation, les associations comprenant plus de vingt personnes, les étrangers 
se rassemblent, plus facilement sous la Restauration que sous la monarchie de Juillet. Certains groupements, relevant de la franc-maçonnerie ou de la charbonnerie, sont clandestins. Le passage à l'action ouverte se fait avec la Jeune Italie de Mazzini, la Société démocratique polonaise et la Ligue des justes, allemande, qui militent sur les plans politique, culturel et éducatif. Certaines associations encouragent l'engagement armé en faveur de leur cause nationale; beaucoup prennent aussi part à la révolution parisienne de 1830 qui s'inscrit pour eux dans le combat pour la liberté du monde.
Delphine Diaz livre sur tous ces points des développements précis, nourris de nombreuses archives et témoignages contemporains. Les aspects culturels, la presse, la vie privée, l'intégration des réfugiés sont abordés très marginalement. Mais l'auteur précise bien qu'elle veut croiser deux approches : les dispositifs de I'accueil et les formes de l'engagement des réfugiés. Dans ces deux domaines, les objectifs sont bien atteints et l'ouvrage offre une mise au point précieuse et souvent neuve.

Ralph Schor

Professeur émérite d'histoire Université de Nice-Sophia-Antipolis 